\title{
User-friendly Interface for Introducing Fuzzy Criteria into Expressive Searches
}

\author{
Mohammad Halim Deedar and Susana Muños Hernández \\ Campus de Montegancedo s/n, Escuela Técnica Superior de Ingenieros Informáticos, \\ Universidad Politécnica de Madrid, Boadilla del Monte 28660, Spain \\ halim.deedarlalumnos.upm.es, susanalfi.upm.es
}

\begin{abstract}
We present a framework that allows any user (without the need of neither technical no theoretical knowledge) to define fuzzy criteria based on the non-fuzzy information stored in databases in an easy way. The interests for developing such a framework is to provide a human-oriented (fuzzy and nonfuzzy) search engine with a user-friendly interface to perform expressive and flexible searches over databases. We achieved this task by providing an intelligent interface for the users to define fuzzy criteria without having any knowledge about its low-level syntax or implementation details. Our framework allows users to pose different queries (combining crisp and fuzzy search criteria) over various conventional and modern data formats such as JSON, SQL, Prolog, CSV, XLS and XLSX. We believe our approach adds to the advancement for more intelligent and human-oriented fuzzy search engines.
\end{abstract}

Keywords: Fuzzy Logic, Fuzzy criteria, Search Engine, Framework.

\section{Introduction}

In general, there is a gap between the database users devoted to access and query conventional and modern databases. Usually, for the expert users it is not a big deal to query different types of databases (conventional and moderns), and they can create and pose standard queries for retrieving information from databases, but the regular users they are only able to access and query the simple and the most conventional databases, and they miss to experience querying over the modern databases. But what all the users (expert and non-expert ones) as a human being have in common is their expressive thoughts and their desire to query in a flexible (fuzzy) way.

It is not practical to store fuzzy information in a database. For example, a database containing the average menu price of restaurants and the user query "I am looking for a cheap restaurant", in this situation the database does not contain any information about "cheap", just the "price-average of restaurants", therefore, it needs a mapping between the "average menu price" and the meaning of "cheap", and this knowledge must be stored somewhere. Considering these points, we tried to remove the gap between the modern databases and the non-technical users by presenting a framework that allows them to create and pose his/her expressive (fuzzy) queries over different conventional and modern database formats (JSON, SQL, Prolog, CSV, XLS, and 
XLSX). For representing an expressive and fuzzy knowledge a bi-valued logic with values "true" and "false", or "yes" and "no" is not enough, therefore the criteria created in our framework is based on Fuzzy Logic (FL) [1], where it does not only indicate if an individual is a member of a set or not, but it also defines a membership degree ( degree of belonging) for the individual of a set. Supposing a restaurant database as shown in Table.1 with the definition for the function "traditional" (Figure 1) related to years since the opening of restaurants and the question "Is there any traditional restaurant?". With FL we can deduce that Zalacain is "very" traditional, museo_del_jamon is "almost" traditional, Kenzo is "hardly" traditional, and Tapasbar is "not" traditional. We highlight the words "very", "almost", "hardly" and "not" because the usual answers for the query are "1", "0.3","0.1" and "0" for the individuals Zalacain, museo_del_jamon, Kenzo and Tapasbar.

Table 1. Restaurant database.

\begin{tabular}{lll}
\hline Name & Years_since_opening & Food_type \\
\hline Zalacain & 45 & Basque \\
Museo_del_jamon & 15 & Spanish \\
Kenzo & 5 & Japanese \\
Tapasbar & 3 & Spanish \\
& & \\
\hline
\end{tabular}

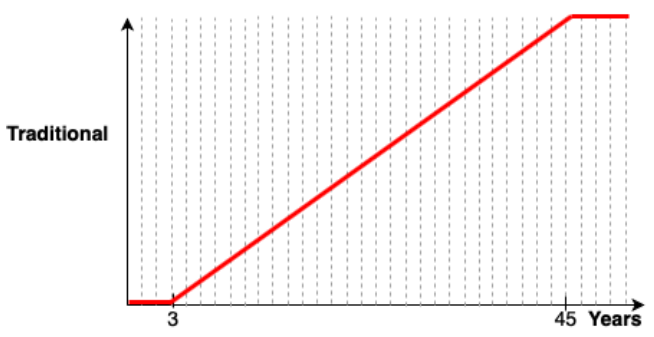

Fig. 2. Traditional fuzzification function.

There are developed tools and theoretical works devoted on posing fuzzy queries over a non-fuzzy databases and obtaining fuzzy information as the ones Bosc and Pivert [2], the SQLf language, Dubois, and Prade [3], Vojtas [4], and the ones with implementations such as Prolog-ELF system [5], the FRIL Prolog system [6], the FProlog language [7], the Fuzzy logic programming environment for research (FLOPER) [8], the fuzzy prolog system [9][10], and RFuzzy [11]. In general, all of them somehow implement the fuzzy set theory introduced by Lotfi Zadeh in 1965 [12]. They have tried to enhance the capability of the existing procedures by proposing new syntactic constructions and providing conversions between the nonfuzzy values needed to execute the query and the fuzzy values in the query. Most of them provided their syntax similar to SQL syntax which is easy to understand and use by the developers and researchers, but difficult for regular users to understand and 
their frameworks are limited only for some specific data formats such as Prolog. What makes our framework different from them is:

(1) Our framework allows any user (expert and non-expert) to define fuzzy criteria for expressive fuzzy searches without the need of knowing about the low-level syntax of the fuzzy functions.

(2) It is the first intelligent framework that allows any users to pose fuzzy and nonfuzzy queries over multiple modern and conventional database formats such as JSON, SQL, Prolog, CSV, XLS and XLSX with a user-friendly querying interface.

(3) The fuzzy criteria created by the users are based on the RFuzzy syntax and semantics which is the approach that provides the most straightforward syntax for creating fuzzy criteria. One of the reasons which makes RFuzzy easier to understand is the use of real numbers instead of intervals between real numbers to represent truth values. The framework takes care of combining between crisp and fuzzy predicates in searching criteria and it manages the introduction of truth value values.

This paper is structured as follows: A brief details about the syntax and semantics of Fuzzy criteria defined in our approach are given in Section 2. We present the details about implementation of our framework (web application) including defining, querying, and modification of Fuzzy criteria, and the results in Section 3. Finally, in Section 4 we give our conclusions.

\section{Syntax and Semantics of Fuzzy criteria}

Before discussing the syntax and semantics of Fuzzy criteria of our approach we include a brief detail about the RFuzzy general rule syntax (Subsection.2.1), and the database syntax (Subsection.2.2) because by some means they are related to each other.

\subsection{General RFuzzy rule syntax}

The general structure that is used to define RFuzzy rules according to a multi-adjoint logic semantics is shown in Def. 1[13].

$$
\begin{aligned}
& P\left(\operatorname{args} P_{j}, V_{j}\right) \stackrel{\left(P r_{j}, V_{c j}\right) \&_{i}}{\longleftarrow} @_{j}\left(Q_{1}\left(\operatorname{args} Q_{1 j}, V_{1 j}\right)\right), \\
& \left(Q_{n}\left(\operatorname{args} Q_{n j}, V_{n j}\right)\right)
\end{aligned}
$$

Where $P$ is the predicate, $j$ is one of the definitions from a set of definitions $j \in[1, N]$ (where $N$ is the number of rules that we have to define for predicate $P$, and $j$ identifies one of these rules). $\operatorname{args} P_{j}$ are the arguments of $P$ in the rule $j$, in the same way, $\operatorname{args} Q_{i}$ are the arguments of $Q_{i}$ where $i \in[1, n]$ and $n$ is the number of elements of the body of the clause. $V_{i}$ is the truth value of the $Q_{i} Q_{i}\left(\operatorname{args} Q_{i}, V_{i}\right)$. @ $@_{j}$ is the 
aggregation operator of the rule $j . V_{c j}$ is the credibility to calculate the truth value, and $P r_{j}$ is the priority of $j$ rule with respect to other rules of $P$ definition.

\subsection{Database syntax}

Before performing any query, the user should upload his/her data files, of course, containing non-fuzzy (crisp) data in any of the accepted formats (JSON, SQL, CSV, Prolog, XLS, XLSX), and after that our framework converts the database into a configuration file (Prolog) including all the necessary headers information that makes that database readable. This configuration file can store the fuzzy criteria created by the user along with the database contents.

The syntax which is responsible for outlining the contents of a database into concepts that we can use in our searches is shown in Def. 2. Where $P$ is the name of the database table, $A$ is its arity, $N$ is the name assigned to a column (field) of the database table where values are of type $T, i \in[1, A]$ identifies each field of the table. We give an example in Eq.3, to elucidate, that the film (name of the table of the database) has four columns, the first one is for the name assigned to each film, the second is for the year in which the film was released, the third is for duration of the films in minutes, and the last one is for the genre of each film whose value belongs to an enumerate range (comedy, thriller, drama, romantic, adventures, etc.).

$$
\begin{aligned}
& \text { define_database }\left(P / A,\left[\left(N_{i}, T_{i}\right)\right]\right) . \\
& \text { define_database }(f i l m / 4, \\
& {[(\text { film_name,string_type }),} \\
& \text { (release_year,integer_type }), \\
& \text { (duration_in_minutes, integer_type }), \\
& (\text { genre,enum_type })]) .
\end{aligned}
$$

The web interface and setters/getters obtain plenty of information from the database definition:

- It gives a value for the query field "individual" $P$ (film).

- It provides a list of values for non-fuzzy predicates (nfp) such as "film name", "release year", "duration in minutes", and "genre" and their types (string_type, integer_type, integer_type, enum_type), which means that in comparison operand field $(c)$, it can be "is equal to " and "is different from" if it of string_type, "is equal to", "is different from" and " is similar to" if it is of enum type or " is equal to", "is different from", "is bigger than", "is lower than", "is bigger than or equal to " and "is lower than or equal to" if it is of integer_type.

- There is a setter/getter for each column, therefore, for example in Definition.3 we get the predicates genre easily $(\mathrm{F}, \mathrm{g})$ and duration_in_minutes $(\mathrm{F}$, dim) that obtain in their second argument the respective value in the database column for the film $F$. 


\subsection{Defining fuzzy criteria syntax}

The syntax presented in Definition. 4 is responsible for defining fuzzifications, and it computes the fuzzy values for fuzzy predicates from the non-fuzzy value existed in some columns in a database, for more details refer to [13]. In the syntax $P$ and $\mathrm{Ni}$ mean the same as in Definition. 2, fPredName is the name for the fuzzy predicate, [valIn, valOut] is a list of pairs of values to define the domain of the fuzzification. To clarify the syntax, we give an example in Eq.5, in which we compute "if a film is new or not from the number of years since its released".

$$
\begin{aligned}
& \text { fPredName }(T): \sim \text { function }\left(P\left(N_{i}\right),[(\text { valin, valOut })]\right) . \\
& \text { new }(\text { film }): \text { function(release_year }(\text { film }),[(1972,0)],[(2018,1)]) .
\end{aligned}
$$

\subsection{Syntax for defining default values for fuzzy criteria}

The syntax presented in Def. 6 is responsible for defining default values for the fuzzy predicates. Its main objective is not to stop a derivation process even if a value is missing. In other words, when the user has not defined a domain value for the fuzzy predicate, in this case, the system will derive the default value defined for that particular predicate. In the syntax the $T$ is same as in Definition. 2 and fPredName is the same as in Def. $4, T V$ is the truth value (a float number in [0,1]) we assign for the default value. We give an example in Def. 7 , in which we say that in the absence of information such as the release year of a film we consider that film will not be new (this is what the zero-value means). The formal semantics for this RFuzzy syntax is shown in Def. 8 , where $p$ is the priority, $v$ is the credibility, $\&_{i}$ is the aggregation operator, and COND is a bi-valued condition. At Definition. 6 we are considering priority $0(p)$, the aggregation operator product $\&_{i}$ the truth value of credibility $1(v)$, and the condition true (COND). Once this structure is defined, the web interface will add them to the list of available predicates for the field $f p$ (see Eq.1) so that the interface will be able to compute default values for the fuzzy predicates.

$$
\begin{aligned}
& \text { fPredName }(T): \sim \text { default_to }(T V) \\
& \text { new }(\text { film }): \text { default_to }(0) . \\
& \text { fPredName } \stackrel{(p, v) \varepsilon_{i}}{\longleftarrow} \text { TV if COND. }
\end{aligned}
$$

\subsection{Fuzzy query syntax}

The query syntax presented in Def.9 [14] shows the structure through which the search engine in approach gets the user's queries as input.

I am looking for a/an

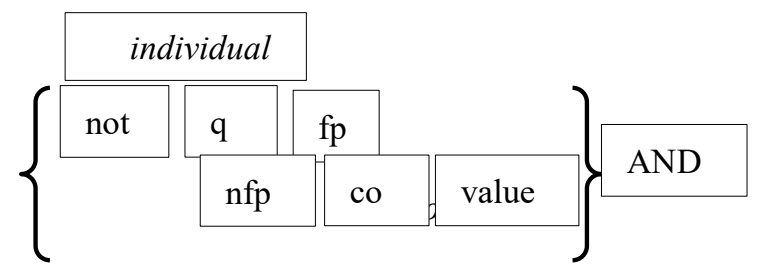


Where individual denotes the element we are looking for, such as (restaurant, cinema, car, etc.), not give a negation mechanism to the queries, $q$ is the quantifier (quite, rather, very, etc.), $f p$ indicates fuzzy predicates such as (cheap, large, close to the city center, etc.), $n f p$ is the non-fuzzy predicates like (price, size, distance to the city center, etc.), co indicates comparison operand and it is consists of operand such as (is equal to, is different from, is bigger than, is lower than, is bigger than or equal to, is lower than or equal to, and is similar to), value asks for a crisp value when the users want to use the comparison operand option such as (is equal to, is different from, or is similar to). The $A N D$ option lets the user add more options and conditions to the query to combine more than one search criterion. Some examples to illustrate the syntax are: "I am looking for a film not very long" (Definition 10), "I am looking for a film whose genre is romantic, and hours of duration is lower than 50 minutes" (Definition 11).

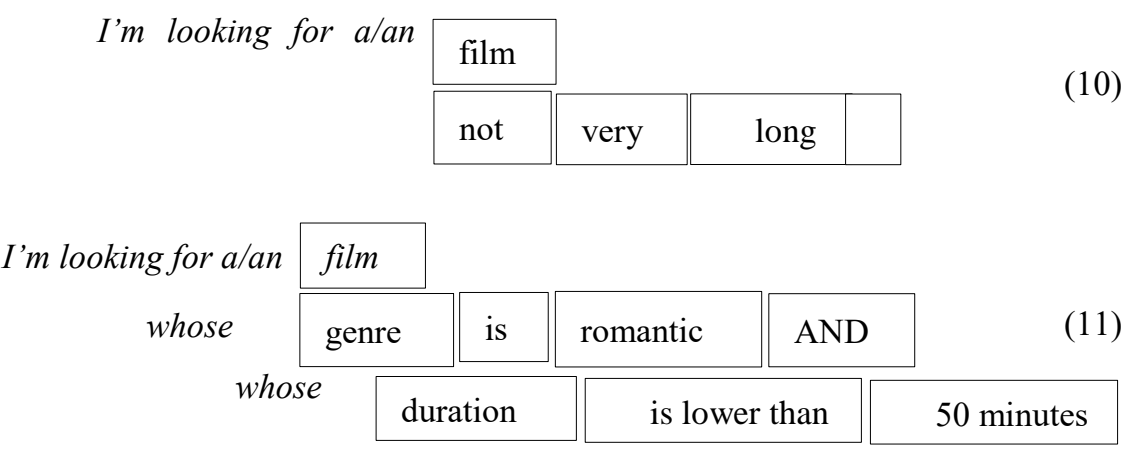

\section{Implementation Details}

Before discussing about the implementation details of our approach, we provide a brief introduction about FleSe (The former version of our approach).

\subsection{FleSe}

FleSe is a tool (web application) for users to perform flexible queries using fuzzy and non- fuzzy search criteria over databases containing crisp information. FleSe uses RFuzzy package which is a Prolog library developed for Ciao Prolog [15]. FleSe uses Fuzzy logic in a Prolog implementation, therefore, we have used this mechanism to perform the fuzzification of FleSe searching criteria. FleSe semantics structure is based on multi-adjoint algebra, for more details refer [16]. 
Our approach enlarges the former FleSe framework [14] with allowing any user to create their fuzzy criteria straightforwardly through an intelligent and smart interface developed mainly in JAVA, JavaScript, and HTML. It let them pose their expressive (fuzzy) queries over various modern and conventional database formats such as JSON, SQL, CSV, Prolog, XLS and XLSX (not like the former FleSe that only allowed Prolog databases).

\subsection{Uploading database interface}

Before defining the criteria, the user has to upload the database in any of the formats (JSON, SQL, CSV, Prolog, XLS, and XLSX). The framework is adaptable therefore other database formats can easily contribute with it. We present the uploading interface in Figure 2 in which we can see a list of databases of various formats uploaded into FleSe. The framework provides a friendly-interface for allowing the users to define the types for the existing data in the database file as we can see in Figure 3 the cars database and the assigned data-types by the user for each column. After assigning the data-types, the interface will prepare a ready configuration file (Prolog file) as shown in Figure 4 out of the uploaded database, and the quantifiers (rather, very, little) will get automatically created and added to the configuration file, and it will be ready to store the criteria created by the users.

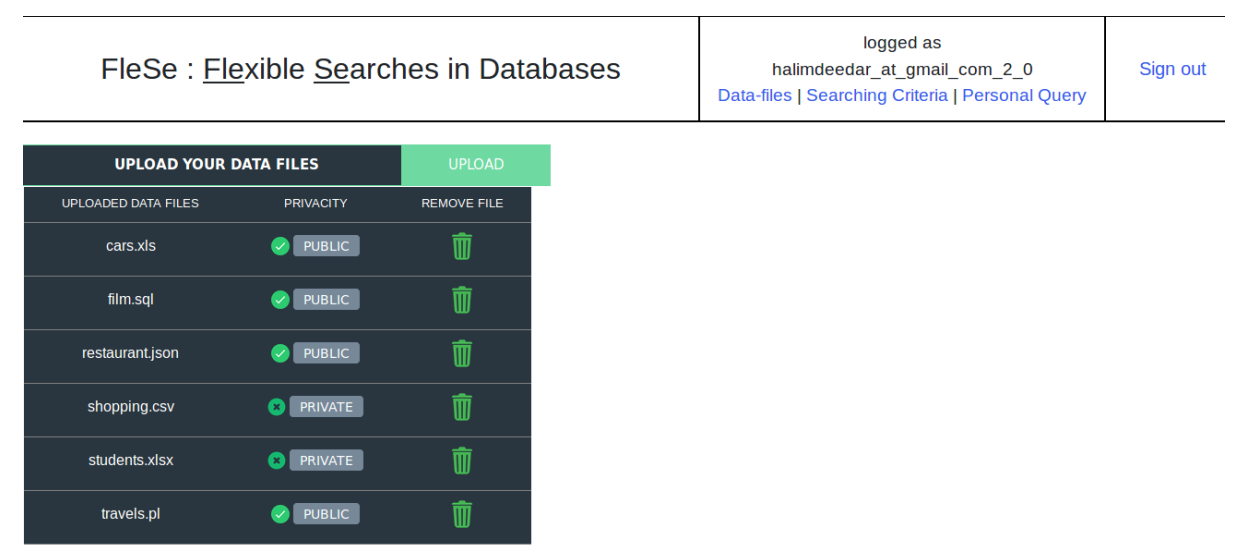

Fig.2. Database uploading interface. 


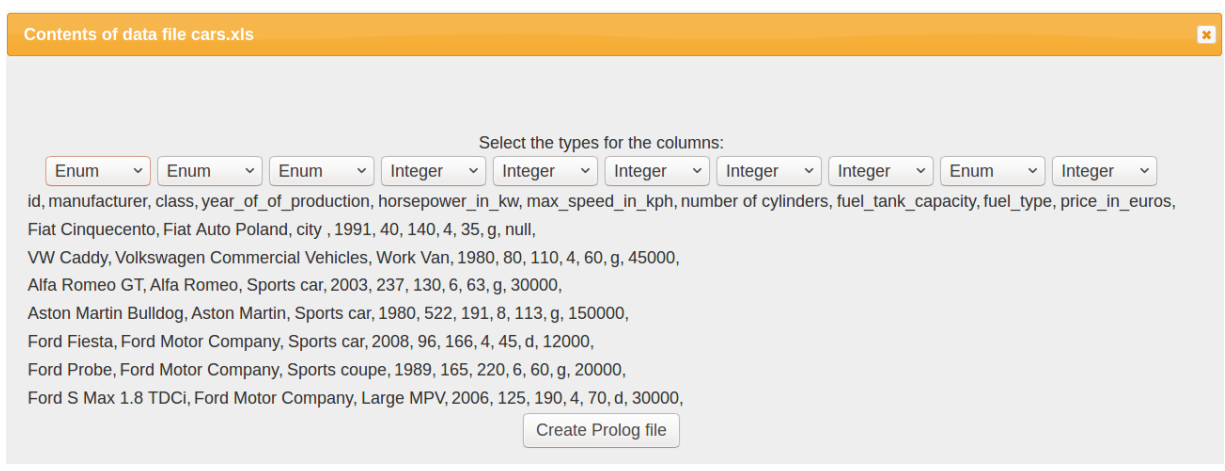

Fig.3. Assigning data types for the columns.

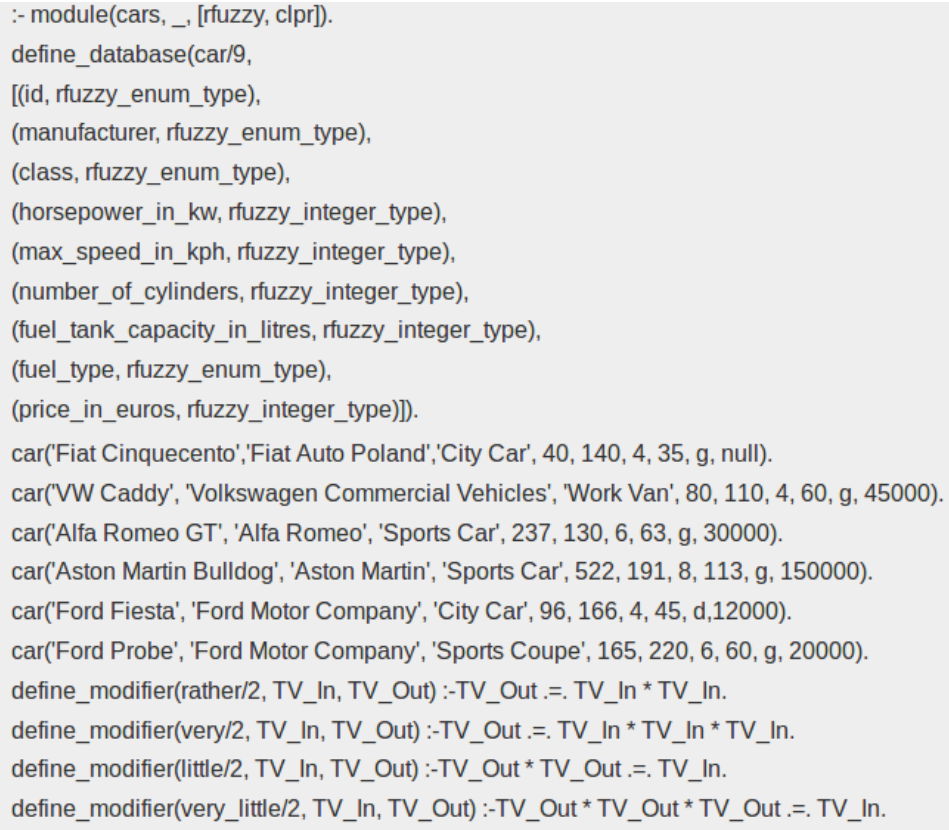

Fig.4. Contents of Prolog data-file.

We can see in (Figure 4), that the contents of our configuration file are made up of four main parts. The first part is the header (:-module(database_name'_' [rfuzzy,clpr]), this header will include the packages and libraries from RFuzzy and CLPR (Constraint Logic Programming) that helps us to introduce the structure of our fuzzy criteria to the search engine while posing an expressive query. The second part is the definition of the columns of the table along with their data-types as explained in Definitions 2, 3. The third part of the configuration file contains the crisp data for our database table, and the fourth part contains the fuzzy criteria and the quantifiers defined by the user. 


\subsection{Interface for adding fuzzy criteria}

We provide an interface (Figure 5)for defining fuzzy criteria in FleSe. This platform allows users to define fuzzy criteria for posing expressive query over databases. The interface allows any user (expert and non-expert ones) to create fuzzy criteria (cheap, expensive, far, etc.) and place them inside the configuration file in an effortless way without being concerned about the syntactical construction of the fuzzy criteria.

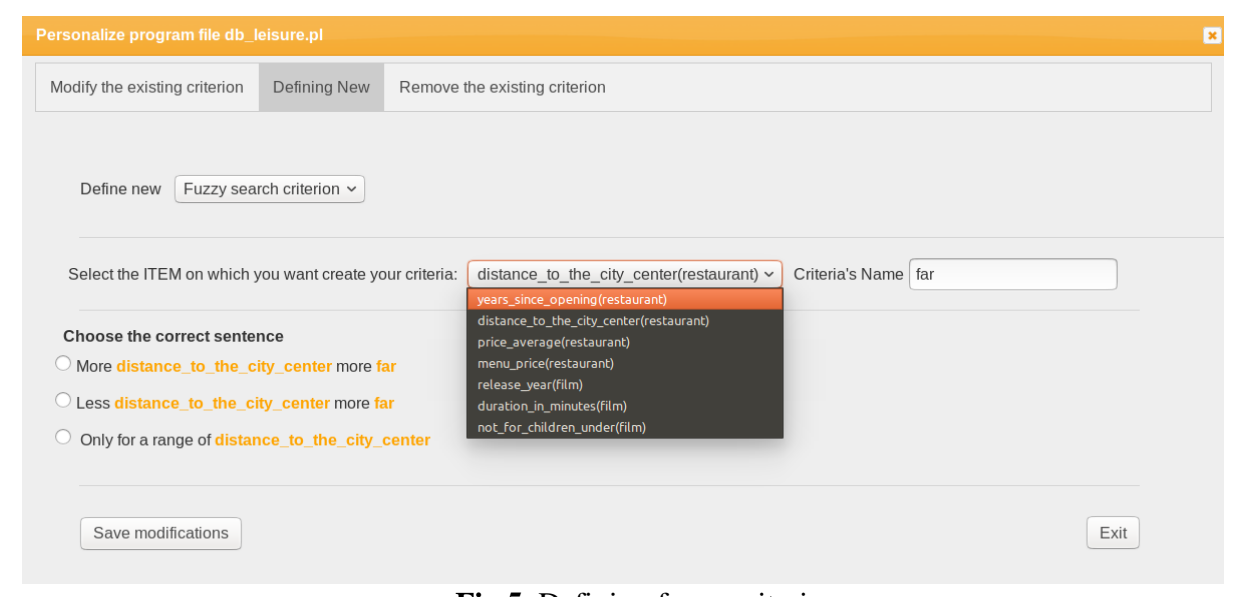

Fig.5. Defining fuzzy criteria.

As we can see in Figure 5, the defining interface for fuzzy search criteria contains a combo-box having a list of the database columns and asking us "to select the ITEM on which you want to create your criteria." And a text-field asking for the name of our fuzzy criteria, and the three radio buttons asking us to choose the format of our fuzzy function (increasing, decreasing or medium). Considered formats are explained in the following Sub-section.

\subsection{Fuzzy Criteria Formats}

While creating the fuzzy criteria, the interface will ask the user to choose the format of the criterion he/she wants to create. The three formats that we consider more common to represent any fuzzy searching criteria are:

Increasing format: A fuzzy criterion is of an increasing format when its value increases as the data item value increases. Taking an example, if the user wants to define a fuzzy criterion for "far" (from the city center) fuzzification function for the query like "I am looking for a restaurant far from the city center", thus the interface provides him the three format options: (1) more distance_to_city_center more far. (2) less distance_to_city_center more far. (3) only for a range of distance to city center. As we know, as much the value of the distance increases between the restaurant and the city center the restaurant will get farther from the city center, that means our Fuzzy criteria is of an increasing format function; therefore, the option (1) is the correct one to select. After selecting the format, the interface asks for the values of the function breakpoints. In case of increasing format the structure of the criteria is [(V1, 
$0),(\mathrm{V} 2,1)]$, in which we just need to assign the two points value (V1 and V2) where $V 1$ value asks for "the maximum value in which the restaurant is not far at all", and the $V 2$ value asks for "the minimum value in which the restaurant is completely far". We illustrate the creation of fuzzy criteria far which is of an increasing format in Figure 6, and the increasing graph for the far fuzzification function in Figure 7.

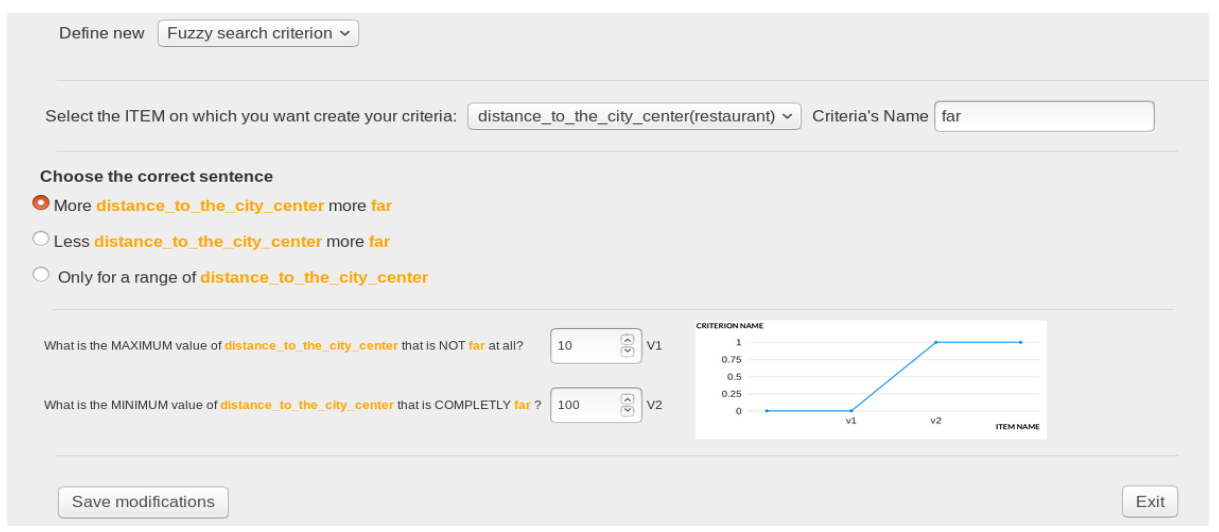

Fig.6. Defining far fuzzification function.

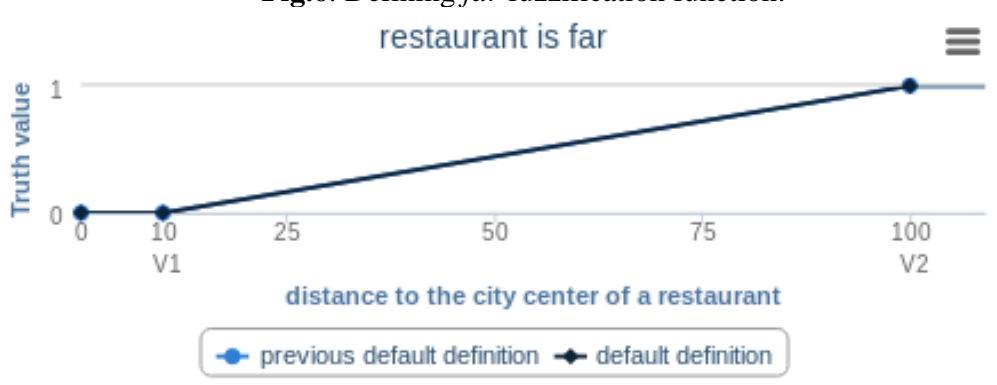

Fig.7. Increasing function format for far fuzzification criteria.

Decreasing format: A fuzzy criterion is of a decreasing format when its value increases as the data item's value decrease. Taking an example, "I am looking for a cheap restaurant", in this case, by decreasing the value of the price_average (which is a column in the restaurant database) the restaurant becomes cheaper; therefore, we select the second option provided by the interface saying, "less price_average more cheap". The decreasing format criteria is made up of the points $[(\mathrm{V} 1, \overline{1}),(\mathrm{V} 2,0)]$, in which we give the value for the two points (V1, and V2) where V1 asks for "the maximum value in which price_average is completely cheap", and V2 asks for "the minimum value in which price_average in is not cheap". We present the decreasing graph for the cheap fuzzification function in Figure 8. 


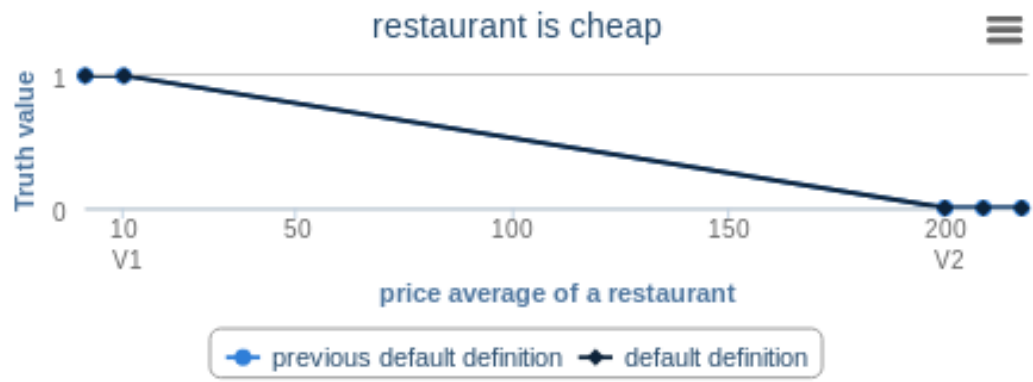

Fig.8. Decreasing function format for cheap fuzzification criteria.

Medium format: A fuzzy criterion is of a Medium format when its value lies between a range of values of the database items. Taking an example, "I am looking for a medium price restaurant". In this case, we will select the third (last) option of fuzzy formats which provides the medium format criteria with the points $[(\mathrm{V} 1,0)$, $(\mathrm{V} 2,1),(\mathrm{V} 3,1),(\mathrm{V} 4,0)]$. Therefore, the interface provides us four different breakpoints (V1, V2, V3, V4) asking for the values to get as an input from the user for defining the criteria in which $V 1$ asks for "the maximum value in which price_average is not medium at all", $V 2$ asks for "the minimum value in which price_average is completely medium", $V 3$ asks for "the maximum value in which price_average is completely medium", and V4 asks for "the minimum value in which price_average is not medium". We present the decreasing graph for the medium_price fuzzification function in Figure 9.

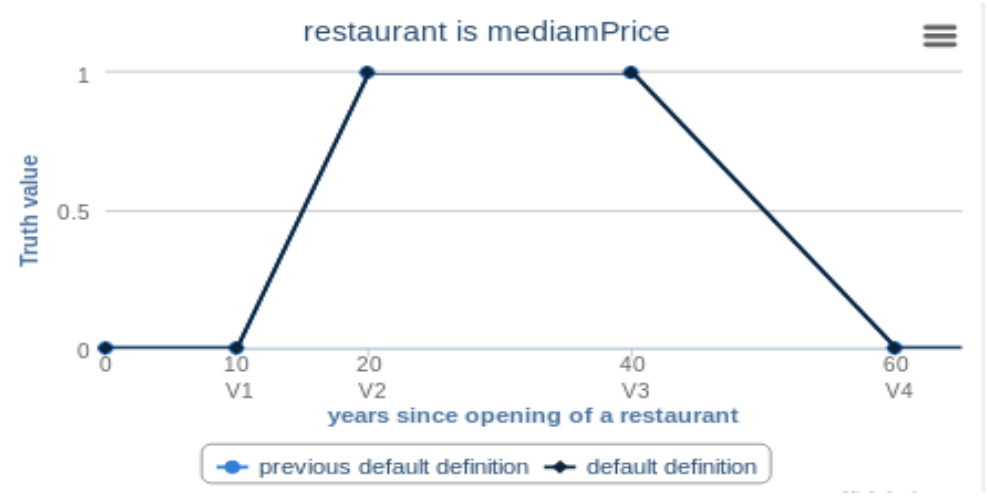

Fig.9. Medium function format for medium_price fuzzification criteria.

After defining the fuzzy search criteria, each particular criterion respectively will get created inside the configuration file as shown in Eqns. 12, 13 and 14.

far(restaruant): function(price_average(restaurant), $[(10,0),(100,1)])$. 
cheap (restaruant): function(price_average(restaurant), $[(10,1),(200,0)])$.

cheap(restaruant): function(price_average(restaurant), $[(10,0),(20,1)$,

$(40,1),(60,0)])$. (14)

We have considered three different formats (increasing, decreasing and medium) for modeling the structure of the potential fuzzy criteria. This will lead us to get functions easily defined by the breakpoints of the domain of fuzzification ([valIn, ValOut]). Where valIn includes the function breakpoints (V1, V2, V3, and V4) that gets its value as an input by the user and valOut holds the truth value ( 0 or 1$)$ which is going to be assigned automatically by the framework based on the type of the fuzzy criteria.

\subsection{Modification interface for fuzzy search criteria}

Once the user has created the criteria, it gets stored in the configuration file (prolog). Therefore, we provide the facility to the users to modify (or edit) the criteria in case if he/she wants to bring changes to the points (V1, V2, V3, and V4). The modification interface is developed in Java, JavaScript, and HTML and it is intelligent enough to take care of the formats of the criteria during the modification process, and it will not allow the user to modify the values for a wrong format of fuzzy criteria, for example, if we want to modify the fuzzy criteria "far" which is of format increasing that follows the rules such as the value for the points "V1 should always be greater than V2" $(\mathrm{V} 1>\mathrm{V} 2)$, and the truth value assigned for V1 should always be "0", and for V2 the truth value should be "1", therefore, if any user wants to modify the criteria far the interface won't allow him/her to give the value V2 greater than V1 or change the truth values. In case if he/she wants to change the format, then we provide them the option to remove the whole criteria and create it again. We present an example in Figure 10 of the form to modify the fuzzy criteria "far" which has been defined in Eq. 12 with the new points $[(20,0),(150,1)]$.

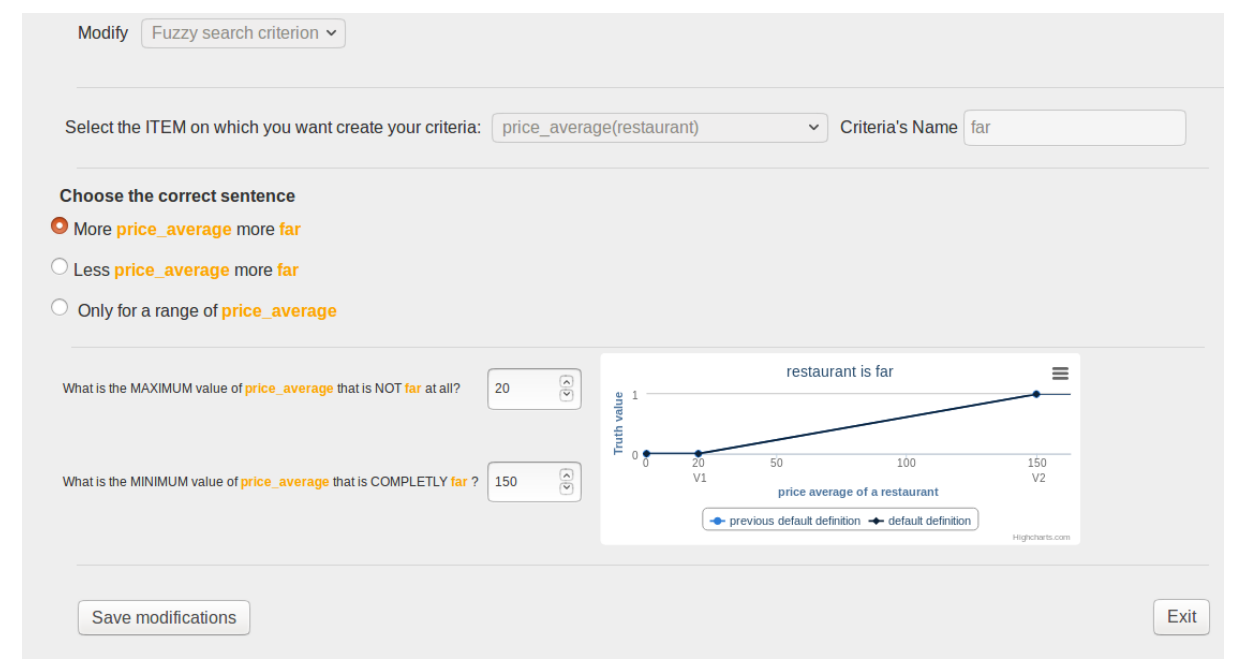

Fig.10. Modification of far fuzzy criteria. 


\subsection{Query interface}

The query interface is a web interface, developed using Java, JavaScript, and HTML. Ajax is used to improve the interface usability through which we can communicate asynchronously with the user web navigator and the server, and it let us use open authentication. The query interface is very flexible, and it allows any user to pose compound queries. To clarify, we give an example of creating a compound query in Figure 11 for the query "I am looking for a little modern, not very long, and a rather funny movie". A compound query could be made by joining crisp and fuzzy search criteria. Suppose if a user wants to pose according to two different searching criteria over a film database such as the first one "I am looking for a comedy movie", and the second one "I am looking for a very new released movie". We can see that in the structure of the first part of the query there is a crisp query and by posing it he/she will get the proper result from the database (if the information is available). In the second part of the query, we can see the fuzzy predicate "new" that is a fuzzy concept, and by posing it, the user will get the proper result. Therefore, the query interface of our approach allows the users to combine both the queries (crisp and fuzzy) to act as a compound query which makes the query more expressive and effective. We give an example of combining crisp and fuzzy queries in Figure 12, for the query "I am looking for a very new comedy movie".

Your query: I'm looking for a

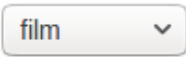

\begin{tabular}{|c|c|c|c|c|c|}
\hline genre & & & $=v$ & comedy & $\checkmark$ \\
\hline$\cdots \vee \vee$ & little & $\checkmark$ & modern & $\checkmark$ & \\
\hline not $\vee$ & very & $\checkmark$ & long durati & $\checkmark$ & \\
\hline$\ldots \vee$ & rather & $\checkmark$ & funny & $v$ & \\
\hline
\end{tabular}

Search

Fig.11. Compound query.

Your query: I'm looking for a

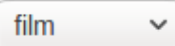

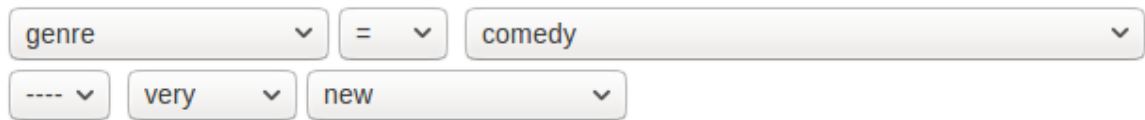

Search

Fig.12. Combining crisp and fuzzy queries. 


\subsection{Output interface}

The output interface provides different sets of results for the query in different tabs (10 best results, results over $70 \%$, results over $50 \%$, results over $0 \%$, and all the results).

The tabs provide different choices to the users to select the one which satisfies his/her needs.

- $\quad$ The tab with 10 best results provides us the 10 best items (or less depends on the number of items existed in the database).

- The tab with the results over $70 \%$ provides us more number of options than the previous tab (10 best results), with the fuzzification degrees between range of $(0.8$ to 1$)$.

- The tab with the results over $50 \%$ provides us the items with the fuzzification degrees between range of ( 0.6 to 1 ).

- The tab with the results over $0 \%$ provides us the items with the fuzzification degrees between range of ( 0.1 to 1$)$.

- And the tab with all results provide us all the items with the fuzzification degree from 0 to 1 that includes the items.

Ajax has been used for getting the result form each tab to avoid waste of computing time.

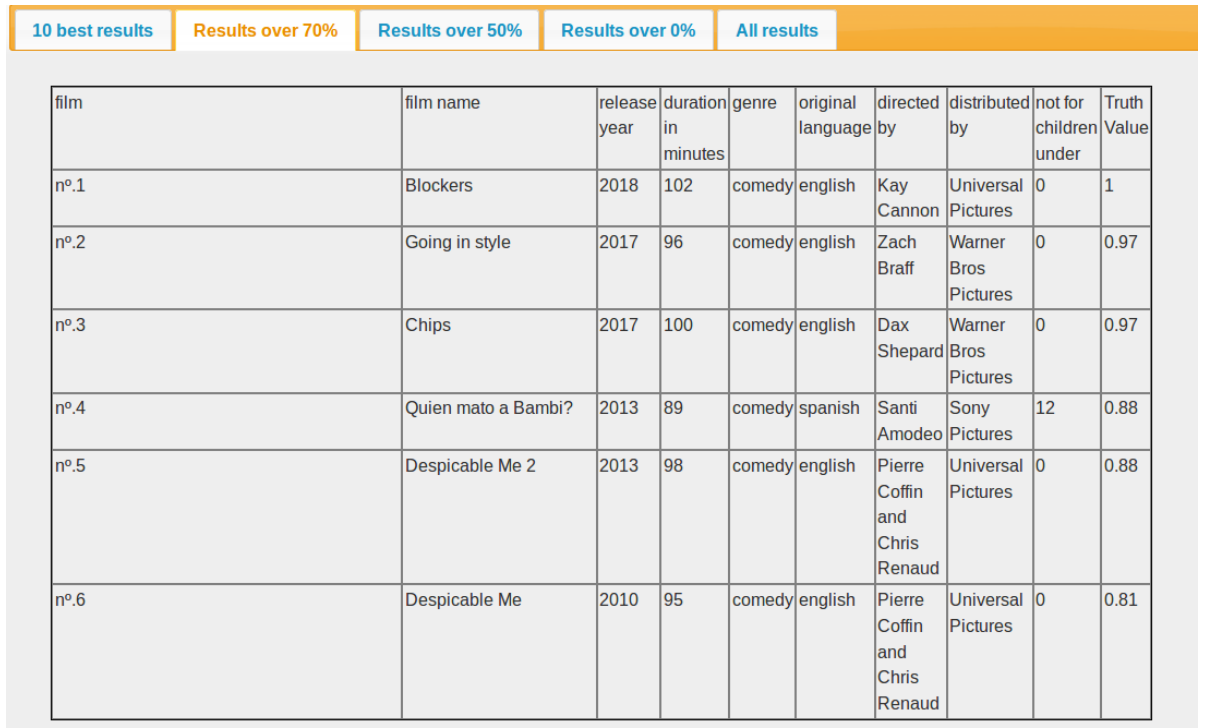

Fig.13. Query results.

We have asked the system for providing us the new films from the films database. Figure 13 shows the result of our query. The criterion of being new is defined over a crisp characteristic of the film that is the release year of the film (shown in Figure 12). 
From the different tags that are offered by the output interface, we have chosen the one that is showing the $70 \%$ best results, that is why the results that we have retrieved they all are having the truth value more than 0.7 . And the best result based on our query is the first one with truth value " 1 ", which indicates the film "Blockers" is the newest released film among the rest of the films which exists in our database.

\section{Conclusions}

We have presented a very practical web interface that allows users to model fuzzy criteria to pose expressive queries over various conventional and modern data formats. It is connected with a search engine developed in RFuzzy. Our contributions to the enlargement of the FleSe framework include:

(1) allowing users to pose their expressive (fuzzy) queries not only over a single data format but also over various modern and conventional data formats such as (JSON, SQL, Prolog, CSV, XLX, XLS).

(2) providing users an intelligent and user-friendly interface that allows them to define, modify and remove the fuzzy search criteria in an easy way without the needs to know about the low-level syntax of the criteria which are not easy for the regular users to understand.

(3) We provide the fuzzy criteria with three different fixed function structure (increasing, decreasing, and medium) to avoid weird types of functions for the fuzzy criteria. It leads to have more explicit and understanding criteria.

We believe our approach will contribute to the development of more intelligent and human-oriented search engines.

Future work will be the extension of the framework on allowing users to search over databases devoted to the definition of similarity relation in a straightforward way.

\section{References}

1. L.A, Zadeh, "Fuzzy Logic," Computer, 21, 4 (1988), 83-93. DO: http://dx.doi.org/10.1109/2.53

2. P. Bosc and O. Pivert, "Sqlf: a relational database language for fuzzy querying," Fuzzy Systems, IEEE Transactions on, vol. 3, no. 1, pp. 1-17, feb 1995.

3. D. Dubois and H. Prade, "Using fuzzy sets in flexible querying: why and how?," In Andreasen, T., Christiansen, H. and Larsen, H. L., editors, Flexible query answering systems, pages 45-60. Norwell, MA, USA (1997).

4. P. Vojtáš, "Fuzzy logic programming," Fuzzy Sets and Systems 124, 3 (2001), 361-370.

5. M. Ishizuka and N. Kanai, "Prolog-elf incorporating fuzzy logic," in IJCAI'85: Proceedings of the 9th international joint conference on Artificial intelligence. San Francisco, CA, USA: Morgan Kaufmann Publishers Inc., 1985, pp. 701-703

6. J. F. Baldwin, T. P. Martin, and B. W. Pilsworth, Fril- Fuzzy and Evidential Reasoning in Artificial Intelligence. New York, NY, USA: John Wiley \& Sons, Inc., 1995.

7. D. Li and D. Liu, A fuzzy Prolog database system. New York, NY, USA: John Wiley \& Sons, Inc., 1990. 
8. P. J. Morcillo and G. Moreno, "Floper, a fuzzy logic programming environment for research," in Proceedings of VIII Jornadas sobre Programacion y Lenguajes (PROLE'08), F. U. de Oviedo, Ed., Gij' on, Spain, october 2008, pp. 259-263.

9. C. Vaucheret, S. Guadarrama, and S. Muñoz-Hernández, "Fuzzy prolog: A simple general implementation using CLP(R)," in LPAR, ser. Lecture Notes in Artificial Intelligence, M. Baaz and A. Voronkov, Eds., vol. 2514. Springer, 2002, pp. 450- 464.

10. S. Guardarrama, S. Muñoz-Hernández, and C. Vaucheret, "Fuzzy Prolog: a new approach using soft constraints propagation," Fuzzy Sets and Systems 144, 1 (2004), 127-150.

11. S. Muñoz-Hernández, V. Pablos-Ceruelo, and H. Strass, "RFuzzy: Syntax, Semantics and Implementation Details of a Simple and Expressive Fuzzy Tool over Prolog," Information Sciences 181, 10 (2011), 1951 - 1970.

12. L.A. Zadeh, "Fuzzy sets," Information and Control 8, 3 (1965), 338 - 353. DOI:

13. V. Pablos-Ceruelo and S. Muñoz-Hernández, "Getting Answers to Fuzzy and Flexible Searches by Easy Modeling of Real-world Knowledge," In: IJCCI 2013 - Proceedings of the $5^{\text {th }}$ International Joint Conference on Computational Intelligence (2013).

14. V. Pablos-Ceruelo and S. Muñoz-Hernández.: "FleSe: A Tool for Posing Flexible and Expressive (Fuzzy) Queries to a Regular Database", In: Proceedings. Distributed Computing and Artificial Intelligence, 11th International Conference, pp.157-164 (2014).

15. The CLIP Lab, "The Ciao Prolog Development System WWW Site, http://www.clip.dia.fi.upm.es/Software/Ciao/." [Online]. Available: http: //www.clip.dia.fi.upm.es/Software/Ciao/

16. J. Medina, M. Ojeda-Aciego, and P. Vojtáš, "A Procedural Semantics for Multi-adjoint Logic Programming,” In: Proceedings of Progress in Artificial Intelligence, pp. 290-297. 